\title{
Fetal Alcohol Syndrome
}

National Cancer Institute

\section{Source}

National Cancer Institute. Fetal Alcohol Syndrome. NCI Thesaurus. Code C84713.

A teratogenic disorder observed in a newborn or child of a mother who consumed alcohol during pregnancy. Manifestations of the syndrome include low birth weight, atypical facies, microcephaly, failure to thrive, developmental defects, organ dysfunction, mental deficiencies, poor motor coordination and behavioral problems. 